This document is the accepted manuscript version of the following article:

Driencourt, L., Federspie1, F., Kazazis, D., Tseng, L. T., Frantz, R.,

Ekinci, Y., ... Gallinet, B. (2020). Electrically tunable multicolored filter using birefringent plasmonic resonators and 1iquid crystals. ACS Photonics,

7 (2), 444-453. https://doi.org/10.1021/acsphotonics.9b01404

\title{
Electrically tunable multicolored filter using birefringent plasmonic resonators and liquid
}

\section{crystals}

Luc Driencourt, ${ }^{\dagger}$ François Federspiel, ${ }^{\ddagger}$ Dimitrios Kazazis, ${ }^{\top}$ Li-Ting Tseng,

Richard Frantz, ${ }^{\ddagger}$ Yasin Ekinci, ${ }^{\top}$ Rolando Ferrini, ${ }^{\dagger}$ and Benjamin Gallinet ${ }^{*} \dagger$

†CSEM Muttenz, Tramstrasse 99, CH-4132 Muttenz

$\ddagger$ Rolic Technologies Ltd., Allschwil, Switzerland

\Paul Scherrer Institut (PSI), Villigen, Switzerland

E-mail: benjamin.gallinet@csem.ch

\begin{abstract}
Dynamic tuning of color filters finds numerous applications including displays or image sensors. Plasmonic resonators are sub-wavelength nanostructures which can tailor the phase, polarization and amplitude of the optical field but they are limited in color vibrancy when used as filters. In this work, birefringence-induced colors of plasmonic resonators and a fast switching thin liquid crystal cell are combined in a multicolored electrically tunable filter. With this mechanism, the color gamut of the plasmonic surface and the liquid crystal cell is mutually enhanced in order to generate all primary additive and subtractive colors with high saturation as well as different tones of white. A single filter is able to cover more than $70 \%$ of the color gamut of standard RGB filters by applying a voltage ranging between $2 \mathrm{~V}$ and $6.5 \mathrm{~V}$. This spectral selectivity is added in transmission without any loss in the image resolution. The presented approach is foreseen to be implemented in a variety of devices including
\end{abstract}


miniature sensors or smart-phone cameras to enhance the color information, ultraflat multispectral imagers, wearable or head--worn displays as well as high resolution display panels.

Keywords: Plasmonic colors, liquid crystals, tunable filters, nanoimprint lithography, color display, multispectral imaging

The digitalization of urban areas, healthcare, homes or agricultural and industrial production has triggered a need for massive deployment of sensing, display and communication devices. Such an increasing amount of information in a reduced volume and cost calls for further miniaturization and integration of multiple functions in optoelectronic systems: it affects in particular consumer smart-phones and wearable displays to airborne detection and industrial control systems. ${ }^{1}$ For example, image acquisition with spectral information is instrumental for industrial quality control, food control, ${ }^{2}$ document security, brand protection or medical diagnostics. ${ }^{3}$ On the other hand, next generation display technologies (augmented and mixed reality glasses, wearables) require optimal use of the space while delivering high resolution pictures. In this context, extensive investigations in the development of filtering technologies have been performed. Pigment-based filters ${ }^{4}$ are limited in their lifetime and in the control of their absorption band. Structural colors generated from the patterning of matter on the length-scale of visible light are not subject to these limitations. ${ }^{5}$ In particular, interference filters can generate a wide variety of saturated colors but with a large number of fabrication process steps, scaling with the number of spectral channels. ${ }^{5}$ Colors induced by resonant nanostructures on the other hand enable patterning of filter sets by variation of a geometrical parameter. ${ }^{5-7}$ Metasurfaces are sub-wavelength nanostructured resonators which can tailor the phase, polarization and amplitude of the optical field to shape the light wavefront. ${ }^{8}$ They have been introduced as a promising approach to generate physical colors. $^{6,9-12}$

In order to overcome the limited amount of spectral and spatial information of conventional filtering approaches, a dynamic change of the filtered color has been extensively 
studied over the last decade: electrically, ${ }^{11}$ thermally, ${ }^{13}$ chemically, magnetically, electrochemically, ${ }^{14-16}$ mechanically ${ }^{17,18}$ or by polarization. ${ }^{19-24}$ The main limitation of chemical processes altering the optical properties of a given material, such as its absorption or refractive index, is their relatively low switching speed compared to standards of image display and acquisition devices. ${ }^{11}$ Liquid crystals, a well-established technology in the display and imaging industries, can translate an electrical stimulus into a variation of the optical properties. The combination of liquid crystals with plasmonic nanostructures and metasurfaces has shown already a great potential, ${ }^{23,25}$ by e.g. a change of refractive index inducing a resonance shift, ${ }^{26-29}$ by polarization rotation, ${ }^{30}$ or controlled orientation of nanoparticles. ${ }^{31}$ However, the obtained gamut suffers from low color vibrancy is still limited to areas below $20 \%$ of the gamut of standard sRGB filters. Recently, the phase retardation induced by birefringent plasmonic resonators has been used to generate larger but incomplete color gamut by mechanical tuning. ${ }^{21,32}$ Fast switching liquid crystal filters based on thin cells are also limited by their spectral width. ${ }^{33,34}$

We propose a strategy for an electrically tunable filter combining the birefringenceinduced colors of plasmonic resonators and a thin liquid crystal cell (LCC). By applying a voltage to the LCC ranging between $2 \mathrm{~V}$ and $6.5 \mathrm{~V}$, a single filter is able to cover more than $70 \%$ of the color gamut of standard sRGB filters, thus exceeding state-of-the-art tunable filters based on liquid crystal technology. In particular, all primary additive and subtractive colors with high saturation as well as different tones of white are generated. In the following, the contribution of the plasmonic resonators to the generated colors is modeled with an harmonic oscillator model and numerical calculations compared with experimental measurements. An electro-optical model is introduced to analyze the contribution and interplay of the different optical elements to the transmitted color. In particular, the enhancement of the plasmonic retardation effect on the spectral bandwidth and color saturation of the LCC is investigated. Finally, a series of experiments illustrates potential applications in display and imaging, with a focus on the selection of entrance polarizer orientation and applied voltages. 


\section{Construction of the color gamut}

Our tunable filter system and its general functioning principle are illustrated in Fig. 1a. The plasmonic nanostructure consists in an array of sub-wavelength and highly anisotropic resonators. Each resonator is a silver nanowire supporting a localized plasmon resonance for the polarization perpendicular to the lines (TM), while weakly interacting for the polarization parallel to the lines (TE). The incident light is linearly polarized at $45^{\circ}$ from the lines. The plasmon resonance for TM polarization yields a transmission dip in amplitude associated with a variation of the phase retardation between TM and TE polarizations. A quarter-wavelength retarding plate, a liquid crystal cell (LCC) and a polarizer are combined in a voltage-controlled polarization analyzer (VCPA). In addition to the rotation of the analyzing polarization, a voltage applied to the LCC results in additional colors from the liquid crystal birefringence. Figure 1b shows the color range in transmission through the system achieved by applying a voltage ranging between $2 \mathrm{~V}$ and $6.5 \mathrm{~V}$. This broad range of color, reaching $70 \%$ of the area of the triangle spanned by RGB filters in the sRGB standard using only a single filter, takes its origin from a mixing of birefringence-induced colors from the plasmonic structure and the LCC. Spectra at a few selected voltages are shown in Fig. 1c-j. In particular, the blue and green colors have high saturation degree. For a voltage of $2 \mathrm{~V}$, the analyzed polarization is $0^{\circ}(\mathrm{TM})$ and the plasmon resonance is probed, leading to a blue color (Fig. 1c). For voltages ranging from $3.15 \mathrm{~V}$ to $3.55 \mathrm{~V}$, the analyzed polarization ranges from $45^{\circ}$ to $60^{\circ}$ with respect to the plasmonic array lines. The resulting color induced by retardation effects in both plasmonic nanostructure and LCC switches rapidly from yellow to red and magenta (Fig. $1 \mathrm{~d}-\mathrm{f}$ ). For a voltage ranging between $3.75 \mathrm{~V}$ and $4.2 \mathrm{~V}$, the analyzed polarization ranges from $80^{\circ}$ to $105^{\circ}$, the light transmitted by the plasmonic nanostructure is mainly color neutral and the resulting color, mainly induced by the LCC, covers the violet and blue (Fig. $1 \mathrm{~g}-\mathrm{h})$. For higher voltages between $5.25 \mathrm{~V}$ and $6.5 \mathrm{~V}$, the cyan color of the plasmonic nanostructure is mixed with the yellow color transmitted by the LCC to generate green (Fig. 1i-j). The peak transmission ranges between $15 \%$ and $45 \%$. 
a

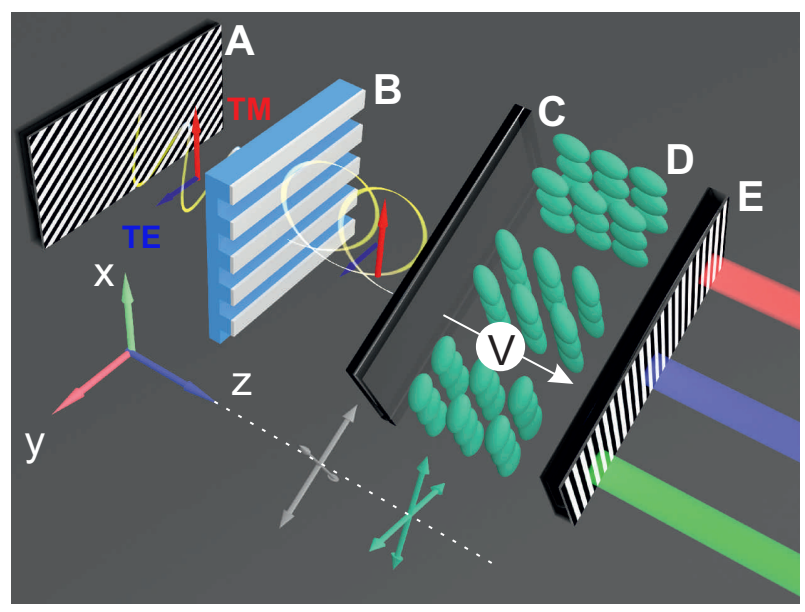

c

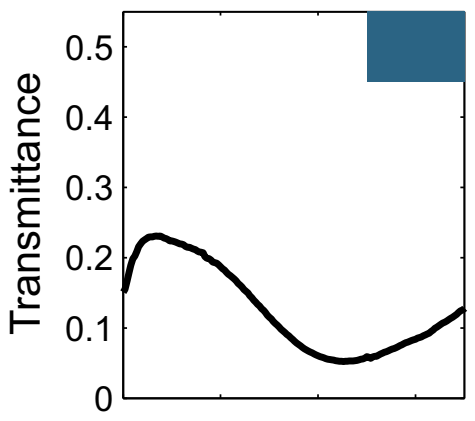

g

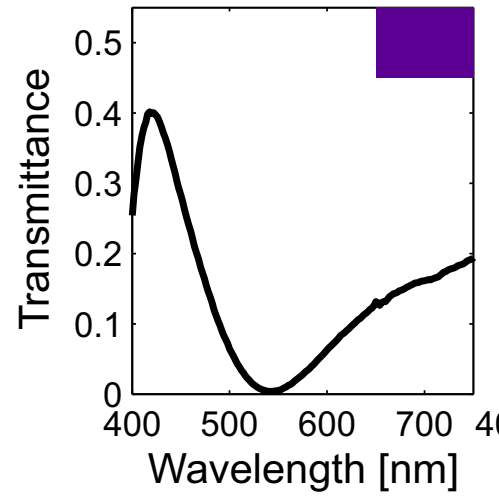

d

$3.15 \mathrm{~V}$

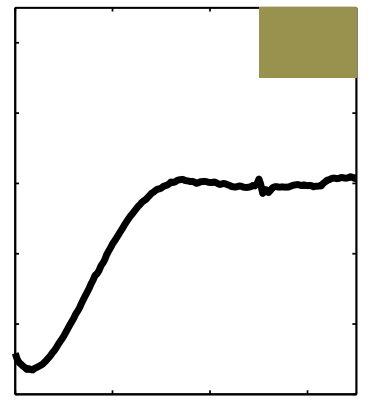

h

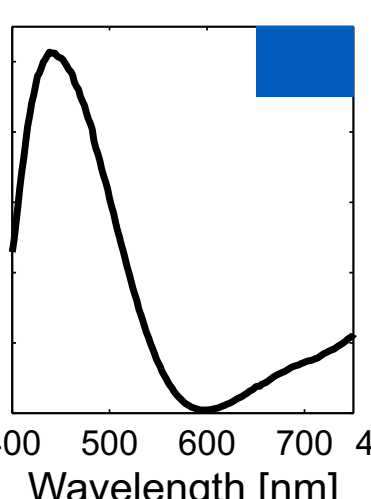

b

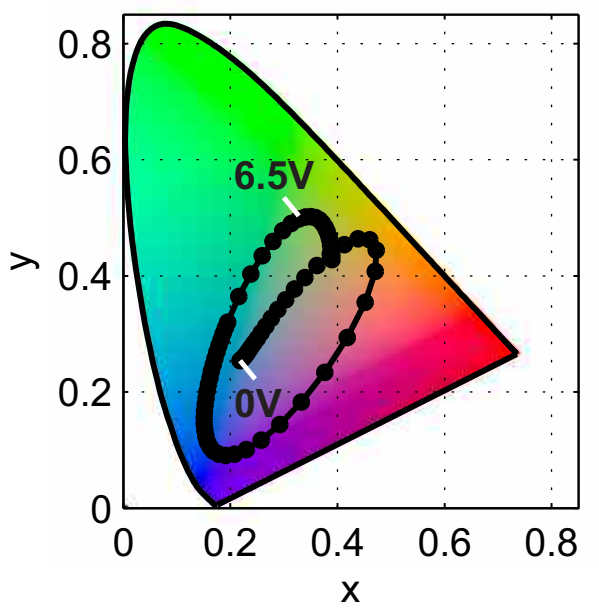

e

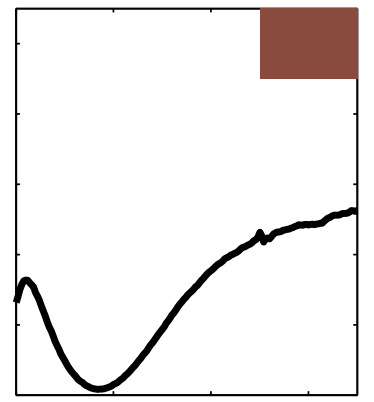

$5.25 \mathrm{~V}$

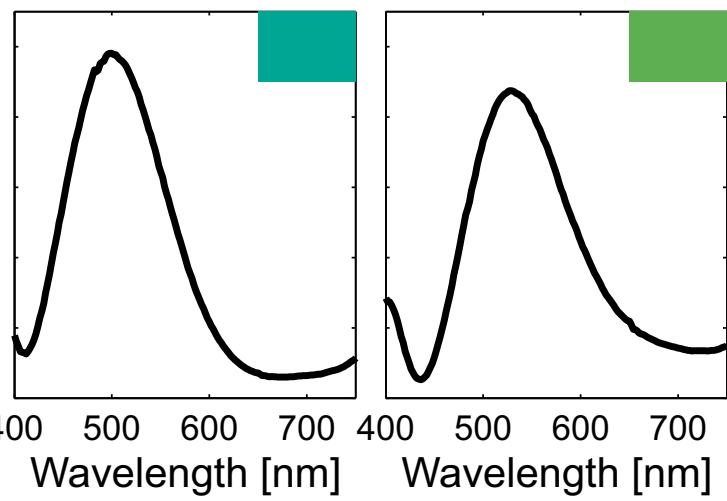

Figure 1: (a) Elements of the filtering system with (A) an entrance polarizer, (B) the plasmonic nanostructures and a voltage-controlled polarization analyzer (VCPA) comprising (C) a quarter waveplate, (D) a liquid crystal cell (LCC) with main retardation axes rotated by $45^{\circ}$ from the main retardation axes of the waveplate, and (E) a polarizer with fixed orientation. The effective orientation of the analyzer is rotated when a voltage is applied to the LCC. TM and TE polarizations are oriented along $x$ and $y$ axis respectively. The plasmonic nanostructures are aligned with the $y$ axis. In order to control the color range transmitted through the system, the polarization analyzer may be rotated with respect to the entrance polarizer and plasmonic nanostructures (here by an angle of $4^{\circ}$ in the $x-y$ plane). (b) CIE color plot of the transmitted colors as a function of the voltage applied to the liquid crystal cell extracted from transmittance measurements. $(\mathrm{c}-\mathrm{j})$ Measured polarized transmittance spectra at selected voltages. The transmission spectra are normalized to the transmission of the input polarizer. 
We now investigate how the birefringent-induced colors from the plasmonic nanostructure and the LCC are mixed and build together the transmitted color. The intensity $I_{\text {out }}$ transmitted through the device as a function of the applied voltage $V$ is the result of contributions from the birefringence of the plasmonic nanostructure and the LCC:

$$
I_{\mathrm{out}}=\frac{\left[T_{\mathrm{TM}} \cos \left(\delta_{V} / 2\right)\right]^{2}}{2}+\frac{\left[T_{\mathrm{TE}} \sin \left(\delta_{V} / 2\right)\right]^{2}}{2}+T_{\mathrm{TM}} T_{\mathrm{TE}} \cos (\Delta \phi) \cos \left(\delta_{V} / 2\right) \sin \left(\delta_{V} / 2\right)
$$

where $\delta_{V}$ is the phase retardation in the LCC induced by the applied voltage, $\Delta \phi$ is the phase retardation induced by the plasmonic nanostructure and $T_{\mathrm{TM}}$ and $T_{\mathrm{TE}}$ are the $\mathrm{TM}-$ and $\mathrm{TE}-$ polarized light spectra transmitted through the nanostructure. We start our discussion with the plasmonic nanostructure. In general, it should be anisotropic to generate different TM and TE responses with a strong phase retardation, and be highly transparent with a stable spectrum with respect to the incidence angle. We also aimed at using an upscalable and robust fabrication process based on UV nanoimprint lithography (UV-NIL), ${ }^{35}$ allowing for the transfer of a nanoscale pattern into a hybrid polymer (Fig. 2). The high resolution imprint master is patterned in silicon by electron beam lithography (EBL) and etching (Fig. 2a). Scanning electron micrographs of the cross section of the master are shown in Fig. 2b. After the UV-NIL step (Fig. 2c), the resulting pattern is coated with silver at an oblique angle in order to have deposition only on the top by self-shadowing. Thus we obtain an array of isolated silver nanowires with the cross section of an inverted U-shape, a pitch of $160 \mathrm{~nm}$ and a width of $110 \mathrm{~nm}$. These parameters have been chosen in order to maximize the color gamut and angular stability (simulations in Supporting Information). This approach is increasing the color saturation as well as the average transmission. Finally, the resulting nanostructure is immediately encapsulated in order to protect it from environmental deterioration. A cross section of the nanostructure before encapsulation is depicted in Fig. 3d.

The orientation of the VCPA as a function of the voltage applied to the LCC has been modelled with Jones matrices (Fig. 2e). The response of the plasmonic nanostructure with 
a
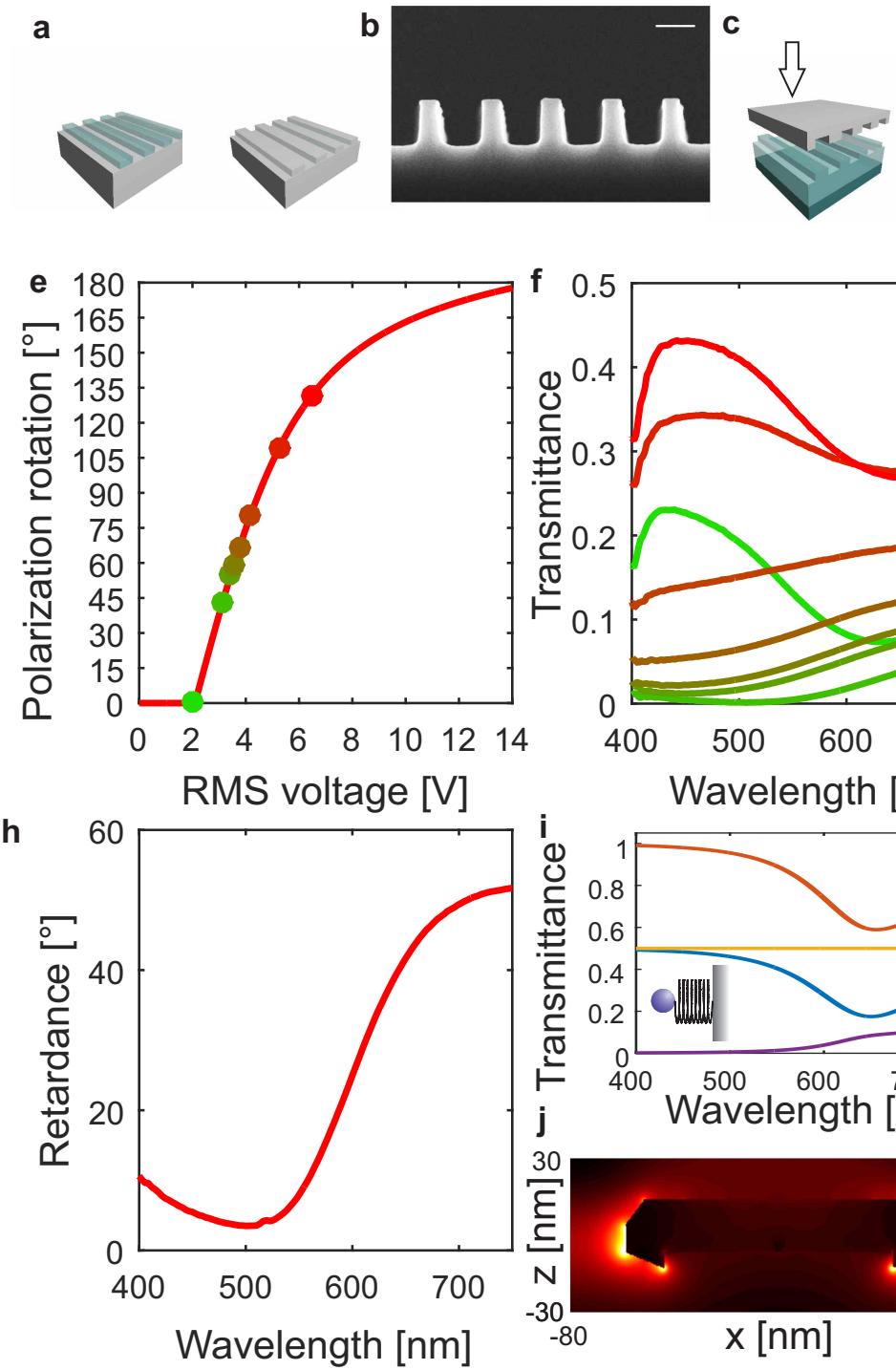
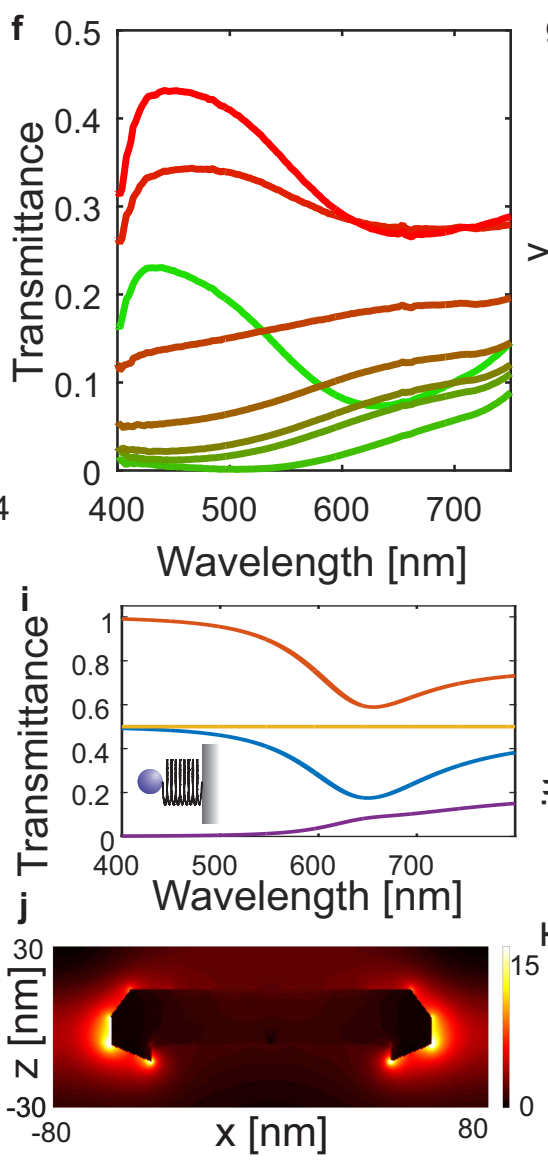
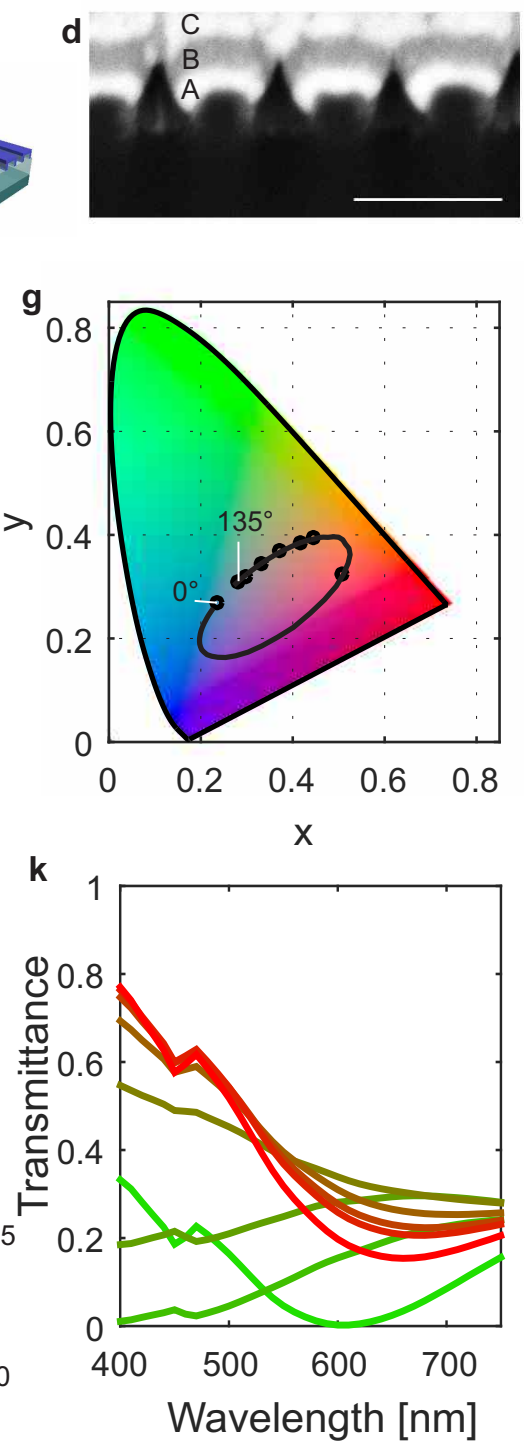

Figure 2: Plasmonic nanostructure as phase retarder. (a) Nanoimprint master generation, using electron beam lithography and transfer to silicon by reactive ion etching. (b) Scanning electron micrograph of the nanoimprint master. Scale bar-100nm. (c) Nanoimprint and thin film coating at oblique deposition angle. (d) Scanning electron micrograph of cross section of the plasmonic nanostructure coated with gold (A) with the same evaporation angle and thickness as the structure of Fig. 1, as well as $\mathrm{Cr}$ (B) and Pt (C) for image contrast and protection during focused ion beam milling. Scale bar-200nm. (e) Model of polarization rotation of the analyzer as a function of the voltage applied to the liquid crystal cell (LCC), with dots showing the voltages used in Fig. 1c-j. (f) Measured transmittance of the plasmonic nanostructure when the LCC-based polarization analyzer is replaced by a mechanically rotated polarizer whose orientation corresponds to the angles defined in panel e, with $0^{\circ}$ corresponding to an electric field polarization oriented along the $y$-axis. (g) Corresponding CIE colors, where the ellipse corresponds to a continuous change of angle from $0^{\circ}$ to $135^{\circ}$. (h) Phase retardation between TM and TE polarizations produced by the plasmonic nanostructure, measured with ellipsometry. (i) Oscillator model showing transmitted

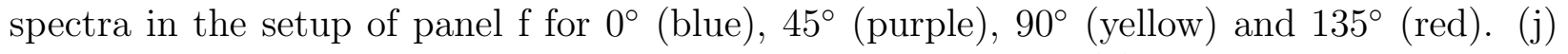
Simulated electric field intensity enhancement in the unit cell. (k) Numerical simulation of the plasmonic nanostructure polarized transmittance for analyzer angles corresponding to panel $\mathrm{f}$. 
a $45^{\circ}$ entrance polarization and a variable output polarization has been measured and normalized to the spectral response of the polarizer (Fig. 2f), with angles corresponding to the different voltages applied to the VCPA. The total amplitude includes the transmission spectrum of the output polarizer, with an average level of $60 \%$. The plasmonic nanostructure is resonant at $620 \mathrm{~nm}$ wavelength and for TM polarization, leading to a dip in transmission for an output polarization angle of $0^{\circ}$. The resulting transmission spectral is modulated with a maximum of around $60 \%$ and a minimum of around 20\%. The output polarization angle of $90^{\circ}$ corresponds to a TE response.

A broad range of colors is generated from the plasmon resonance and its birefringenceinduced interferences (Fig. 2g) using tuning by polarization state, as also reported in previous work as an ellipse in CIE coordinates. ${ }^{21}$ The gamut area amounts to approximately $30 \%$ of the sRGB filter range. When the output polarizer is oriented at $45^{\circ}$ or $135^{\circ}$, the phase retardation is directly contributing to the transmitted intensity by a constructive or destructive interference effect, yielding a red and cyan color, respectively. These two colors, as well as the fact that the plasmonic nanostructure is color neutral for orientations around $90^{\circ}$ are key to the generation of the color gamut when combined with the VCPA, as will be discussed later. A variety of polarization-dependent and retarder structures could be potentially used, ${ }^{19,36}$ provided that their colors are complementary to the VCPA colors. In contrast, a single polarizer and any polarization-dependent resonant structure yields only two colors and their mixing, i.e. a line in CIE coordinates. ${ }^{19,36}$

The phase retardation between TM and TE polarizations has been measured with ellipsometry, showing a phase jump of $50^{\circ}$ around the plasmon resonance (Fig. 2h). The transition between colors depends on the phase and amplitude response of the plasmonic nanostructure. This behaviour can be reproduced in Fig. 2i using a harmonic oscillator model for the TM response. In the Supporting Information, the phenomenological parameters modeling the phase retardation, oscillator amplitude, resonance frequency and losses are studied, showing how they can influence the spectral response. In particular, it is found that 
the saturation of the red color at $45^{\circ}$ depends on the amplitude of the phase retardation. The electric field distribution in Fig. $2 \mathrm{j}$ shows an enhancement at the extremities of the metallic structure for TM polarization (along $x$-axis). It originates from the excitation of a localized surface plasmon and dipolar charge distribution at the interference between the metal and the surrounding dielectric material. Dipolar localized surface plasmon resonances are known for their large angular stability. They are strongly coupled with the radiating field, which as an effect increases the resonance bandwidth. ${ }^{37}$ The effect of the amplitude and phase retardation of the plasmonic nanostructure on the transmitted color is reproduced by numerical simulations in Fig. 2k: the plasmon resonance for $0^{\circ}$ orientation of the output polarizer, the constructive interference in the red for $45^{\circ}$ and the redistribution of intensities for $135^{\circ}$ generating the cyan. A higher transmission in the blue range stems from the spectrally flat response of the analyzer, which is particularly impacting the spectra around $90^{\circ}$. As the periodicity of the array is $160 \mathrm{~nm}$, grating dispersion or diffraction effects are avoided. In addition, the planarity of the plasmonic nanostructure is high, which has the advantage to provide a high angular stability up to $40^{\circ}$ tilt (simulations and measurements in the Supporting Information).

A large variety of colors can be obtained from the plasmonic nanostructure using the rotation of the analyzing polarization (yellow, magenta, red, cyan or blue), except green. This color is particularly relevant for visible light filtering. On the other hand, it is shown in Fig. 3a and e that the LCC alone is able to generate a large color gamut with voltage, which can be understood by considering a wavelength dependence of the coefficient $\delta_{V}$ in Eq. (1). However, a large thickness of liquid crystal molecules is necessary to obtain a complete palette of visible saturated colors, which drastically increases the switching time ${ }^{33,34,38}$ and hinders applications. Here, the thickness of the LCC is less than 7 microns, which yields a good switching time (maximum $75 \pm 10 \mathrm{~ms}$ measured for the highest voltage) but also a high full-width half-maximum (FWHM). ${ }^{34}$ Fig. 3a shows the transmission of the VCPA at different voltages, with FWHM up to $180 \mathrm{~nm}$ at $6.5 \mathrm{~V}$. 

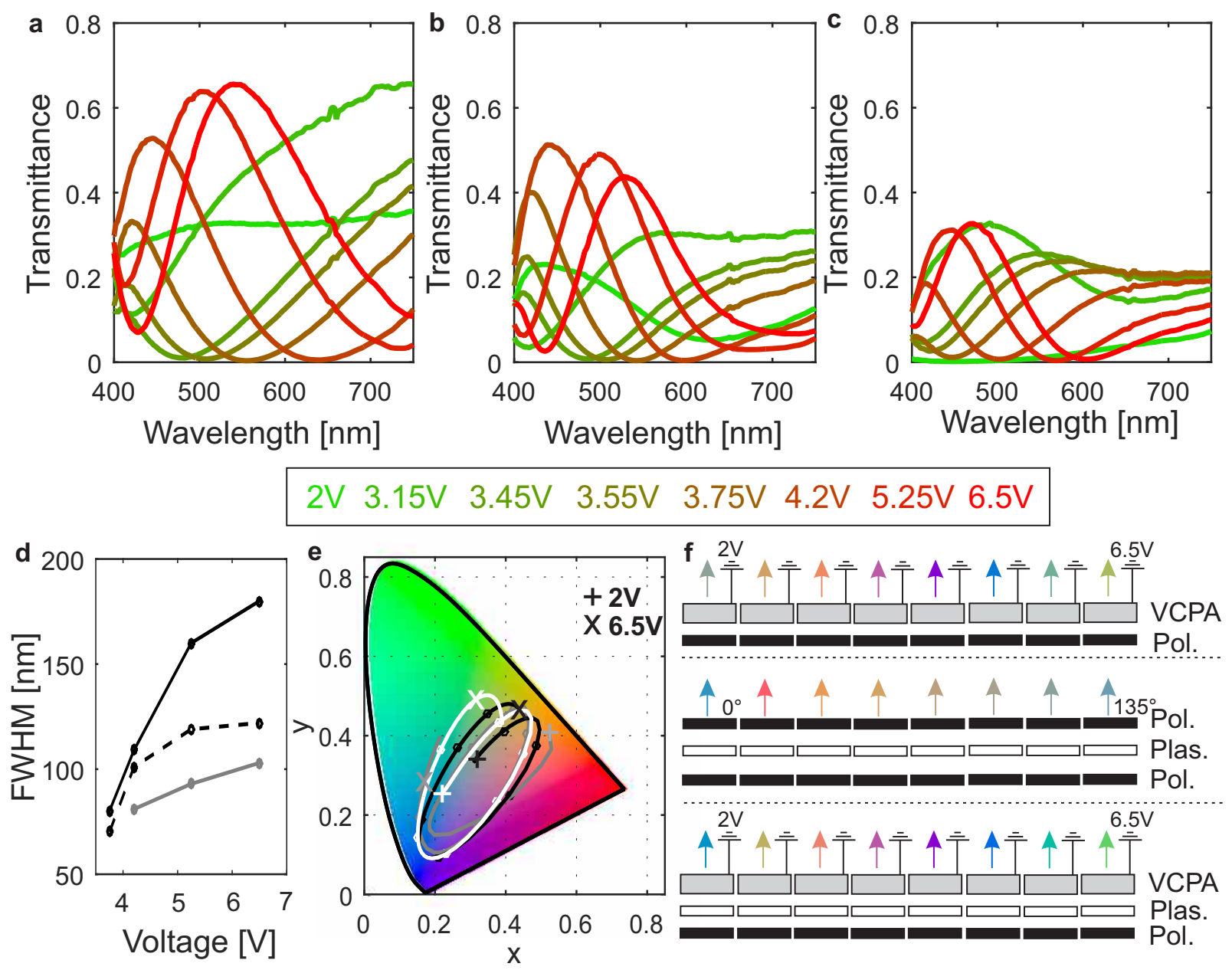

Figure 3: Experimental analysis of color contributions in polarized transmission. (a) Configuration of Fig. 1 without plasmonic nanostructure. (b) With plasmonic nanostructure and $4^{\circ}$ rotation of polarization analyzer. (c) With plasmonic nanostructure and $45^{\circ}$ rotation of polarization analyzer. In $(\mathrm{a}-\mathrm{c})$, the applied voltages are green $-2 \mathrm{~V}$ to red $-6.5 \mathrm{~V}$, with $3.15 \mathrm{~V}, 3.45 \mathrm{~V}, 3.55 \mathrm{~V}, 3.75 \mathrm{~V}, 4.2 \mathrm{~V}$ and $5.25 \mathrm{~V}$ as intermediate values. (d) Full-width half-maximum (FWHM) of transmission peaks as a function of the applied voltage: blackpanel a, dashed-panel b, grey-panel c. (e) Corresponding CIE color plots: black-panel a, white-panel b, grey-panel c. The pseudo-ellipses correspond to a continuous change of voltage. The transmission spectra are normalized to the transmission of the input polarizer. (f) Mechanism of mixing of birefringence-induced colors between the VCPA and plasmonic nanostructure (Plas.) placed between polarizers (Pol.), showing true colors extracted from panel a (top), Fig. 2f (middle) and panel b (bottom). 
In the configuration including the plasmonic nanostructure (Fig. 3b), the VCPA is oriented in such a way that the TM polarization is probed at a voltage of $2 \mathrm{~V}$. Voltages at $2 \mathrm{~V}$ and $4.2 \mathrm{~V}$ yield a blue color despite the fact that they correspond to perpendicular polarizations (Fig. 2e): this is due to the fact that the plasmon resonance excited under TM polarization solely contributes to the transmitted spectrum at $2 \mathrm{~V}$, while at $4.2 \mathrm{~V}$ the LCC mainly contributes to the transmitted color (Fig. 3e). A strong effect is the reduction of FWHM of the transmission peaks at high voltages (Fig. 3d). This is achieved by combining the cyan nanostructure (orientations above $100^{\circ}$ in Fig. 2f) to the birefringence-induced colors of the LCC (transmission spectra above $4 \mathrm{~V}$ in Fig. 3a). As a consequence, the transmission at large wavelengths is reduced and the FWHM reduced. In the Supporting Information, pictures and a video of the fabricated device of lateral dimension $20 \mathrm{~mm}$ as a function of the applied voltage is shown, for the cases without (Fig. 3a) and with the plasmonic nanostructure (Fig. 3b). Figure 3c shows a configuration aimed at enhancing the red. The entrance polarization is rotated by $45^{\circ}$ from the plasmonic nanostructure so that the color is dominated at low voltages by the red of the nanostructure, which is more saturated than the birefringence-induced red of the LCC. From Figure 3d, the effect of FWHM reduction is also reproduced at high voltages because the nanostructure still possess a cyan transmission spectrum between $135^{\circ}$ and $180^{\circ}$ analyzing polarization (Fig. 2f).

Overall, red is obtained from the plasmonic structure, blue from the LCC and green from the combination of cyan and yellow from the plasmonic structure and the LLC, respectively. Other colors such as violet, magenta, yellow, cyan, turquoise and different tones of white are generated at intermediate voltages. The area of gamut in the CIE coordinates covers $70 \%-75 \%$ of the sRGB filter range using this approach (white and grey curves in Fig. 3e). In comparison, previous works report on an area of approximately $15 \%{ }^{27}$ using active liquid crystal tunability and $30 \%$ by polarizer rotation. ${ }^{21}$ A maximal unpolarized transmission of $50 \%$ is allowed by the system due to the polarization selection of the entrance polarizer: after passage through the first polarizer, up to $100 \%$ of the light can be transmitted in cer- 
tain configurations, depending on the phase retarders and output polarizer configuration. The entrance polarizer can also be actively tunable in order to address unpolarized light. We highlight that the birefringence-induced colors of the plasmonic nanostructure and the LCC are complementary and their mixing is the key to achieve a broad color gamut, as sketched in Fig. 3f. Plasmonic nanostructures possess unique birefringence properties compared to liquid crystals, enabling the generation of a broader palette than thin liquid crystal tunable filters, including green. The thin LCC is able to generate a blue with saturation comparable to standard filters at $4.2 \mathrm{~V}$, but lacks other saturated primary colors. A possible way to construct a tunable filter with fully saturated RGB colors could be envisioned as follows: a retarder nanostructure which has saturated red and green for two polarization orientations, and is color neutral for the polarization orientation corresponding to $4.2 \mathrm{~V}$. In the Supporting Information, spectra and colors are reconstructed from polarization-resolved transmission measurements the phase retardation measurement of the plasmonic nanostructure and Eq. (1), thus validating the model.

\section{Display and imaging applications}
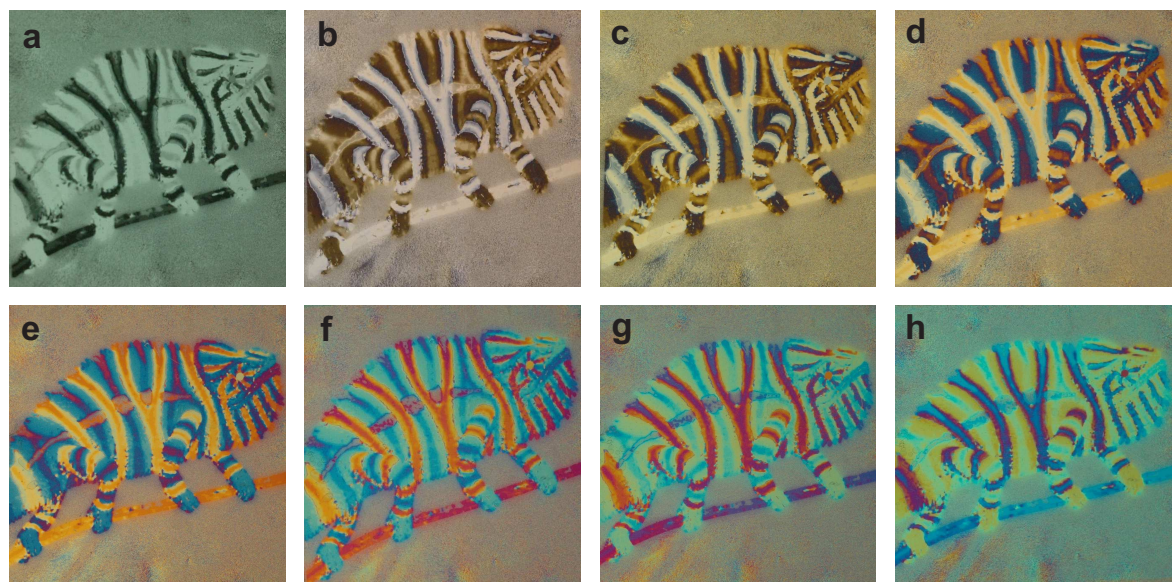

Figure 4: Tunable filter for colored display. The input polarizer is patterned with 18 different orientations in the shape of a chameleon. The polarizing orientation determines the range of accessible colors as well as the voltage at which they appear. (a) 0V (b) $3.2 \mathrm{~V}$ (c) $3.4 \mathrm{~V}(\mathrm{~d}) 3.8 \mathrm{~V}$ (e) $4.3 \mathrm{~V}$ (f) $4.9 \mathrm{~V}$ (g) $6.3 \mathrm{~V}$ (h) $8.5 \mathrm{~V}$. 
In order to illustrate the potential of this approach for displays, a large area device has been realized using an organic light emitting diode (OLED) and an input polarizer (element (A) in Fig. 1a) patterned with different orientations encoded in an image of a chameleon (Fig. 4). The plasmonic nanostructure is identical to Fig. 2. More details on the design and fabrication of the input polarizer are provided in the Supporting Information and the methods section. The plasmonic nanostructure and LCC are homogeneous over the device area. For orientations of $0^{\circ}$ and $90^{\circ}$ (stripes), the field incident on the plasmonic nanostructure is polarized such that only the TE and TM response are observed and the phase retardation effect does not contribute to the colors. For orientations of $45^{\circ}$ and $135^{\circ}$, all colors reported in Fig. 1 are observed (skin of the chameleon), in particular the green color obtained by combining the cyan of the plasmonic nanostructure and the yellow of the LCC. The background contains random orientations of the input polarizer, thus no observable change of color is observed. Deviations from the colors extracted from transmission measurements in the rest of the manuscript originate from the spectral distribution of the OLED and the spectral response of the image sensor used for recording the pictures. In order to build a display device based on the tunable filter, the electrically active layer would have e.g. a backplane equipped with an array of thin film transistors. ${ }^{39}$ The image information would be encoded in the backplane, while the plasmonic nanostructure does not need to be patterned and aligned at pixel scale. The generation of colors inside the ellipse in the CIE plot of Fig. 1a would be performed in a time sequence and the effective color reconstructed by time-averaging. It should also be noted that in addition to the primitive RGB colors, other colors such as white, yellow, orange, magenta, turquoise or cyan can directly be obtained without having to mix together the primitive colors. This property may potentially open opportunities for displays for rendering more natural colors. In addition, since the size of the display is only limited by the pixel resolution, our tunable filter may be used for the miniaturization of wearable displays, including in particular projectors for head-worn displays for virtual and augmented reality. For example, birefringence-induced colors of 
liquid crystals have been used to build a color display in a liquid crystal on silicon (LCOS) reflective spatial light modulator. ${ }^{40}$

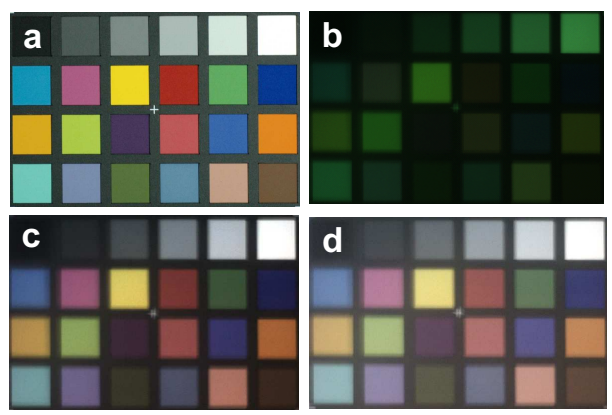

Figure 5: Tunable filter for color imaging. (a) Reference image. (b) Image reconstructed after acquisition at three different voltages, corresponding to blue, red and green. (c) Image after white balance. (d) Image after white balance and gamma correction.

Conversely, our tunable filter can be used for imaging applications. In Fig. 5, a RGB imaging device is built with the tunable filter and a single channel camera. The image of a color checker is recorded by a camera equipped with the tunable filter at different voltages. For a more balanced color distribution, the tunable filter is used in a configuration where the plasmonic nanostructure is rotated by $45^{\circ}$ from the analyzer (Supporting Information). The plasmonic nanostructure is identical to Fig. 2. The selected voltages correspond to the blue $(4.4 \mathrm{~V})$, green $(10 \mathrm{~V})$ and red $(3.6 \mathrm{~V})$. A simple image processing consisting of a white balance and gamma correction taking into account the logarithmic response of the human eye (gamma factor 1.6, following e.g. Burgos ${ }^{41}$ allows the construction of the image shown in Fig. 5c. All colors are well reproduced, thus showing that color information is obtained without compromising the image resolution. As all components in the device are planar, they can be integrated on chip for compact vision systems. Alternatively, this approach can be used to miniaturize the imager for the same pixel size, which can be a key advantage in portable or wearable imaging devices. We also envision this device to provide spectral information well beyond the three primary colors without compromising the image resolution by using more voltages and a more elaborate image processing algorithm, for e.g. high resolution multispectral imaging. ${ }^{32,42}$ The orientation of the entrance polarizer can also be 
electrically tuned to generate polarization-resolved imaging.

The ability to generate all available colors in a short time frame is therefore calling for developments in fast switching liquid crystal devices with minimal frequency of $180 \mathrm{~Hz}$, a requirement for typical display applications. Considering that the response time of a LCC is proportional to the square of the cell thickness, ${ }^{33,34}$ the approach presented here allows for a drastic reduction of the cell thickness compared to liquid crystal filters and presents a high potential for high frequency switching and low power consumption. Finally, it could also be applicable to reflective or transflective displays by including multiple transmissions in the different layers. ${ }^{39,40}$

\section{Conclusions}

This Article has reported on an electrically tunable filter with wide color gamut, achieved by the mixing in transmission of birefringent-induced colors from a plasmonic nanostructure and a LCC. A broad variety of colors can be spanned upon application of a voltage in the range between $2 \mathrm{~V}$ and $6.5 \mathrm{~V}$. In particular, red is obtained from the plasmonic structure, blue from the LCC and green from the combination of cyan and yellow from the plasmonic structure and the LLC, respectively. Other colors such as violet, magenta, yellow, cyan, turquoise and different tones of white are generated at intermediate voltages. Overall, an area above $70 \%$ of the standard sRGB filter range is covered with a single tunable filter. Compared to liquid crystal tunable filters, the resonance spectral width of the transmitted light is decreased from $180 \mathrm{~nm}$ to $120 \mathrm{~nm}$ in the green range and the corresponding color saturation greatly improved. The nanostructures support a strong plasmon-induced birefringence, have a peak transmission up to $80 \%$ and an acceptance angle of $90^{\circ}$. The construction of the colors transmitted by the tunable filter has been modeled and validated through a series of optical characterization of the individual elements. Finally, a colored image has been acquired using the filter in combination with a grey level camera. 
The fabrication of the plasmonic nanostructures has been performed by UV nanoimprint technology and silver deposition which is up-scalable for mass manufacturing. ${ }^{43,44}$ The plasmonic nanostructure and the LCC are realized individually and the two components are eventually combined in a final assembly step, thus simplifying future system production at industrial scale. A variety of anisotropic nanostructures could potentially be applicable, including aluminum structures compatible with CMOS processes, ${ }^{10,45}$ hybrid ${ }^{24}$ or dielectric ${ }^{22,36,46}$ structures. The latter structures have the potential for generating low bandwidth, i.e. large color saturation, provided a minimization of the angular acceptance for practical applications. Symmetry-broken structures have the ability to support subradiant modes with low bandwidth. ${ }^{47,48}$ The effect can also be extended to other spectral ranges, such as the near infrared or short-wavelength infrared by adaptation of the structure parameters and materials.

The demonstration of an electrically tunable filter with large color gamut makes a step forward in the miniaturization of display and imaging devices. Its implementation is envisioned in high resolution and high switching speed display panels, reflective or transflective displays, or in miniature projection systems in wearable or head-worn devices. The additional spectral information beyond RGB filters can be used on smart-phone cameras for enhanced color rendering, towards highly integrated and versatile multispectral imaging systems for a broad variety of applications such as industrial or personal quality control, food control $^{2}$ or personal diagnostics. ${ }^{3}$

\section{Acknowledgement}

Fundings from NanoArgovia (grant A12.13) are gratefully acknowledged.

\section{Supporting Information Available}

Supporting information is available free of charge via the Internet at http://pubs.acs.org. 
Oscillator model. Modeling and optimization of resonance-induced colors in the nanostructure. Full system spectral reconstruction. Measurement of transmission of the plasmonic nanostructure as a function of polarization, tilt angle and orientation of the principle axis. Construction of input polarizer with multiple orientations. Filter configuration for image acquisition. Photograph sequences and videos of devices.

\section{Methods}

Fabrication of plasmonic nanostructure The fabrication of the plasmonic nanostructure is based on UV-nanoimprint lithography (UV-NIL). The patterning of the nanoimprint master has been performed with EBL (Raith EBPG 5000+ system operated at $100 \mathrm{keV}$ ) as the nanostructure needs to have low line edge roughness, be mechanically stable, and its height should be on the order of $120 \mathrm{~nm}$. The EBL tool has a low field stitching error of $\sim 20-30 \mathrm{~nm}$ for fields of $320 \mu \mathrm{m}$. A $100 \mathrm{~mm}$ Si wafer was initially covered with $20 \mathrm{~nm}$ of $\mathrm{Cr}$ in a blanket deposition by electron beam evaporation. A thin 100 nm PMMA layer was subsequently spin-coated onto the Cr layer and exposed by EBL. The wafer was developed in an MIBK:IPA 1:3 solution and the pattern was then transferred from the PMMA layer onto the Cr layer by plasma etching of the $\mathrm{Cr}$ (Cl-based plasma). The PMMA layer was subsequently stripped in an acetone bath and the grating pattern was transferred into the Si wafer by inductively coupled plasma reactive ion etching (ICP-RIE) at a very low pressure of 8 mTorr and a temperature of $5^{\circ} \mathrm{C}$. A mixture of $\mathrm{C}_{4} \mathrm{~F}_{8}$ and $\mathrm{SF}_{6}$ was used in a $3: 1$ ratio, which resulted in a well-controlled and anisotropic etch. ${ }^{49}$ Finally the Cr mask was removed by Cl-based plasma etching. The imprint material is Ormocomp from Microresist Technology, on a borofloat glass substrate. A loss of depth of $5 \%$ to $10 \%$ at each generation is expected during imprint, which was considered in the original design and fabrication of the nanoimprint master. Two depositions of silver are performed at an angle of $75^{\circ}$ to the normal of the substrate, calibrated such that the total thickness is $20 \mathrm{~nm}$. The encapsulation 
is performed by drop-casting and curing a thick layer of Ormocomp with a borofloat glass plate on top.

Cell preparation A formulation of photoalignment polymer is spin-coated onto two ITO coated glass substrates. After spin-coating, the substrates are subjected to a baking procedure consisting of pre-baking at $80^{\circ} \mathrm{C}$ and post-baking at $200^{\circ} \mathrm{C}$. Then, the substrates are exposed to p-polarized UVB light having an incidence angle of $40^{\circ}$ respective to the substrate normal. The plane of polarization is parallel to the substrate's shortest edges. The cells are assembled with the two substrates, the exposed polymer layers facing the inside of the cell. The substrates are adjusted relative to each other such that the induced alignment directions are parallel to each other, with a gap of approximately 7 microns. The cells are capillary filled with liquid crystal MLC-6610 (Merck, negative dielectric anisotropy). Finally, after soldering wires to both sides, the filled cells are further subjected to a thermal annealing, thereby completing the cell process. The cells configuration is vertical alignment (VA), which means the liquid crystal molecules are almost perpendicular to the substrates, with a pretilt angle of approximately $88^{\circ}$ in the absence of electric field, thus giving virtually no retardation. When increasing the $(\mathrm{AC})$ voltage, the molecules continuously rotate until being parallel to the substrate, giving more than one full wave of retardation. A quarter waveplate foil (Edmund Optics) is laminated onto one side of the cells, with its fast axis at $45^{\circ}$ compared to the cells' edges. A film polarizer (Edmund Optics) is laminated onto the other side of the cells, with its absorption axis corresponding to the quarter waveplate's fast axis, thus building a polarization analyzer with voltage-controlled orientation. The LCC switching time has been measured between crossed polarizers from 10\% transmission to $90 \%$ RMS for a voltage of $6.5 \mathrm{~V}$ at ambient temperature. The rise and release times have been measured to $45 \pm 8 \mathrm{~ms}$ and $75 \pm 10 \mathrm{~ms}$, respectively.

Fabrication of patterned polarizer A photoalignment material is spin-coated onto a glass substrate and cured before exposure in a direct polarization writing machine. Pixel- 
defined areas are subjected to polarized irradiation using 16 different azimuthal angles, covering a $180^{\circ}$ range. Then, a formulation of dichroic dye and liquid crystal monomer mixture (host-guest system) is spin-coated onto the stack, followed by a final step of annealing and cross-linking with UV-A.

Modeling and measurement of system electro-optical response The electro-optical response of the system is modeled using Jones matrices. The entrance polarizer is placed at $45^{\circ}$ from the polarization axis of the plasmonic nanostructure. The field transmitted by the plasmonic nanostructure is generally described by a Jones vector $T_{\text {in }}$ including a TM-polarized amplitude coefficient $T_{\mathrm{TM}}$, a TE-polarized amplitude coefficient $T_{\mathrm{TE}}$ and a phase delay $\Delta \phi$ between the two polarizations. These responses depend on the wavelength, which is kept implicit in the following for clarity. The system also comprises a quarterwavelength plate, a liquid crystal cell with fast and slow axis rotated by $45^{\circ}$ from the axis of the quarter-wavelength plate, and a polarizer with fixed orientation, represented by their respective matrices $\Lambda_{4}, L$ and $P_{\text {out }}$. The amplitude of the field transmitted through the system is therefore:

$$
\begin{aligned}
T_{\mathrm{out}} & =P_{\mathrm{out}} \cdot L \cdot \Lambda_{4} \cdot T_{\mathrm{in}} \\
& =\left(\begin{array}{ll}
1 & 0 \\
0 & 0
\end{array}\right) \cdot\left(\begin{array}{cc}
\cos \left(\delta_{V} / 2\right) & -i \sin \left(\delta_{V} / 2\right) \\
-i \sin \left(\delta_{V} / 2\right) & \cos \left(\delta_{V} / 2\right)
\end{array}\right) \cdot\left(\begin{array}{cc}
e^{-i \pi / 4} & 0 \\
0 & e^{i \pi / 4}
\end{array}\right) \cdot\left(\begin{array}{c}
\frac{T_{\mathrm{TM}}}{\sqrt{2}} e^{i \Delta \phi} \\
\frac{T_{\mathrm{TE}}}{\sqrt{2}}
\end{array}\right) \\
& =\frac{1}{\sqrt{2}}\left(T_{\mathrm{TM}} e^{i \Delta \phi} \cos \left(\delta_{V} / 2\right)+T_{\mathrm{TE}} \sin \left(\delta_{V} / 2\right)\right)
\end{aligned}
$$

where $\delta_{V}$ is the phase delay induced by the voltage-dependent birefringence $\delta_{V} n(V)$ in the liquid crystal material of thickness $t$ and can be modeled by:

$$
\delta_{V}=\frac{2 \pi \delta_{V} n(V) t}{\lambda}
$$


The intensity of the transmitted field can be simplified to:

$$
\left|T_{\mathrm{out}}\right|^{2}=\frac{\left[T_{\mathrm{TM}} \cos \left(\delta_{V} / 2\right)\right]^{2}}{2}+\frac{\left[T_{\mathrm{TE}} \sin \left(\delta_{V} / 2\right)\right]^{2}}{2}+T_{\mathrm{TM}} T_{\mathrm{TE}} \cos (\Delta \phi) \cos \left(\delta_{V} / 2\right) \sin \left(\delta_{V} / 2\right)
$$

In the case of a linearly polarized input light $T_{\text {in }}=(\cos \theta, \sin \theta)^{\mathrm{T}}$, the system simplifies to a polarization analyzer whose axis depends on the applied voltage: $\left|T_{\text {out }}\right|^{2}=\cos ^{2}\left(\theta-\delta_{V} / 2\right)$. In the Supporting Information, this model is used to fully reconstruct the voltage-dependent spectra of the full system in Fig. 1 from optical measurement of polarized transmission and phase retardation of the nanostructure, together with the birefringence-induced colors of the LCC.

Numerical simulations The numerical simulations of the nanostructure transmission have been performed with the rigorous coupled wave analysis using 60 Fourier harmonics. ${ }^{7}$ The silver permittivity has been extracted from experimental values. ${ }^{50}$ Owing to the surface scattering and grain boundary effects in the thin film, its imaginary part is multiplied by a phenomenological factor of two. The silver structures are completely surrounded by a homogeneous medium with the permittivity of Ormocomp.

Surface integral equation (SIE) with periodic boundary conditions was used to compute the intensity of the near field at the resonant wavelength in the unit cell of the plasmonic nanostructure. ${ }^{51}$ The cross-sectional SEM measurement was used to design the simulated geometry. The transmission spectrum for an incident TM-polarized plane wave was first calculated. The electromagnetic field intensity was then computed at resonance (wavelength of $677 \mathrm{~nm})$ in a cross section of the unit cell.

Optical measurements The retardation spectrum of the sample was measured with an ellipsometer. In the basic transmission system, a variable polarizer converts light from the source to an input beam with a known and adjustable polarization. The output of the variable polarizer is then passed through the sample under study and the output polarization 
determined using a polarization analyzer. The transmission spectra where measured with a spectrometer (SpectraScan PR-735, Photo Research). The elements represented in Fig. 1a were placed between the light source and the spectrometer, such that the light is normally incident to the samples. The intensity transmitted through the input polarizer was used to normalize the spectra. A function generator was used to drive the polarization analyzer with a square waveform of $50 \%$ duty cycle and amplitude varied from $0 \mathrm{~V}_{R M S}$ to $6.5 \mathrm{~V}_{R M S}$. For the imaging experiments of Fig. 5, the device of Fig. 1a was placed in front of a monochrome CCD camera (Baumer TXG14). The object to image (colorChecker, X-Rite) was placed at about $50 \mathrm{~cm}$ to the imaging setup and was illuminated with a LED screen (Dörr LP-400). Three monochrome images were acquired at the voltages corresponding to the primary colors. The reconstruction of the colored image was then performed numerically by using a color map defined by the RGB coordinates of the colors of the tunable filter corresponding to those three voltages.

Structure analysis Scanning electron microscope (SEM) images were obtained with an FEI Helios focused ion beam (FIB) SEM. In order to image the cross section of the plasmonic metasurface before encapsulation, gold was evaporated instead of silver under the same conditions because of the fast oxidation of silver in air. The total thickness evaporated was measured on a glass substrate. A Cr layer was sputtered on the sample prior to SEM imaging in order to have a conducting surface and also to obtain a good contrast with the evaporated gold layer. For the FIB milling, a Pt layer was previously deposited in order to avoid damaging the sample during the milling process.

\section{References}

(1) Photonics21 Multiannual Strategic Roadmap 2021-2027. https://www photonics21. org/download/news/2019/Europes-age-of-light-Photonics-Roadmap-C1.pdf?m= 1552465949\&, Accessed: 2019-04-23. 
(2) Liu, Y.; Pu, H.; Sun, D.-W. Hyperspectral imaging technique for evaluating food quality and safety during various processes: A review of recent applications. Trends Food Sci. Tech. 2017, 69, $25-35$.

(3) Kha, M. J.; Khan, H. S.; Yousaf, A.; Khurshid, K.; A., A. Modern Trends in Hyperspectral Image Analysis: A Review. IEEE Access 2018, 6, 14118-14129.

(4) Nakamura, J. Image Sensors and Signal Processing for Digital Still Camera; CRC Press: Boca Raton, FL, 2006.

(5) England, G. T.; Aizenberg, J. Emerging optical properties from the combination of simple optical effects. Rep. Prog. Phys. 2017, 81, 016402.

(6) Kristensen, A.; Yang, J. K. W.; Bozhevolnyi, S. I.; Link, S.; Nordlander, P.; Halas, N. J.; Mortensen, N. A. Plasmonic colour generation. Nature Reviews Materials 2016, 2, 16088.

(7) Quaranta, G.; Basset, G.; Martin, O. J. F.; Gallinet, B. Recent Advances in Resonant Waveguide Gratings. Laser Photonics Rev. 2018, 12, 1800017.

(8) Arbabi, A.; Horie, Y.; Bagheri, M.; Faraon, A. Dielectric metasurfaces for complete control of phase and polarization with subwavelength spatial resolution and high transmission. Nat. Nanotechnol. 2015, 10, 937-943.

(9) Kumar, K.; Duan, H.; Hegde, R. S.; Koh, S. C. W.; Wei, J. N.; Yang, J. K. W. Lasing action in strongly coupled plasmonic nanocavity arrays. Nat. Nanotechnol. 2012, 7, $557-561$.

(10) Olson, J.; Manjavacas, A.; Basu, T.; Huang, D.; Schlather, A. E.; Zheng, B.; Halas, N. J.; Nordlander, P.; Link, S. High Chromaticity Aluminum Plasmonic Pixels for Active Liquid Crystal Displays. ACS Nano 2016, 10, 1108-1117. 
(11) Shao, L.; Zhuo, X.; Wang, J. Advanced Plasmonic Materials for Dynamic Color Display. Adv. Mater. 2018, 30, 1704338.

(12) Kim, I.; Yoon, G.; Jang, J.; Genevet, P.; Nam, K. T.; Rho, J. Outfitting Next Generation Displays with Optical Metasurfaces. ACS Photonics 2018, 5, 3876-3895.

(13) Shu, F.-Z.; Yu, F.-F.; Peng, R.-W.; Zhu, Y.-Y.; Xiong, B.; Fan, R.-H.; Wang, Z.-H.; Liu, Y.; Wang, M. Dynamic Plasmonic Color Generation Based on Phase Transition of Vanadium Dioxide. Adv. Opt. Mater. 2018, 6, 1700939.

(14) Wang, G.; Chen, X.; Liu, S.; Wong, C.; Chu, S. Mechanical Chameleon through Dynamic Real-Time Plasmonic Tuning. ACS Nano 2016, 10, 1788-1794.

(15) Jiang, N.; Zhuo, X.; Wang, J. Active Plasmonics: Principles, Structures, and Applications. Chem. Rev. 2018, 118, 3054-3099.

(16) Atighilorestani, M.; Jiang, H.; Kaminska, B. Electrochromic-Polymer-Based Switchable Plasmonic Color Devices Using Surface-Relief Nanostructure Pixels. Adv. Opt. Mater. 2018, 6, 1801179.

(17) Lütolf, F.; Casari, D.; Gallinet, B. Low-Cost and Large-Area Strain Sensors Based on Plasmonic Fano Resonances. Adv. Opt. Mat. 2016, 4, 715-721.

(18) Tseng, M. L.; Yang, J.; Semmlinger, M.; Zhang, C.; Nordlander, P.; Halas, N. J. Two-Dimensional Active Tuning of an Aluminum Plasmonic Array for Full-Spectrum Response. Nano Lett. 2017, 17, 6034-6039.

(19) Ellenbogen, T.; Seo, K.; Crozier, K. B. Chromatic Plasmonic Polarizers for Active Visible Color Filtering and Polarimetry. Nano Lett. 2012, 12, 1026-1031.

(20) Park, C.-H.; Yoon, Y.-T.; Shrestha, V. R.; Park, C.-S.; Lee, S.-S.; Kim, E.-S. Electrically tunable color filter based on a polarization-tailored nano-photonic dichroic resonator featuring an asymmetric subwavelength grating. Opt. Express 21, 28783-28793. 
(21) Duempelmann, L.; Luu-Dinh, A.; Gallinet, B.; Novotny, L. Four-Fold Color Filter Based on Plasmonic Phase Retarder. ACS Photonics 2016, 3, 190-196.

(22) Nagasaki, Y.; Suzuki, M.; Takahara, J. All-Dielectric Dual-Color Pixel with Subwavelength Resolution. Nano Lett. 2017, 17, 7500-7506.

(23) Kim, M.; Kim, I.; Jang, J.; Lee, D.; Nam, K. T.; Rho, J. Active Color Control in a Metasurface by Polarization Rotation. Applied Sciences 2018, 8, 982.

(24) Zhao, Z.-J.; Lee, M.; Kang, H.; Hwang, S.; Jeon, S.; Park, N.; Park, S.-H.; Jeong, J.-H. Eight Inch Wafer-Scale Flexible Polarization-Dependent Color Filters with Ag-TiO2 Composite Nanowires. ACS App. Mat. Inter. 2018, 10, 9188-9196.

(25) Ma, Z.; Meng, X.; Liu, X.; Si, G.; Liu, Y. J. Liquid Crystal Enabled Dynamic Nanodevices. Nanomaterials 2018, 8, 982.

(26) Franklin, D.; Chen, Y.; Vazquez-Guardado, A.; Modak, S.; Boroumand, J.; Xu, D.; Wu, S.-T.; Chanda, D. Polarization-independent actively tunable colour generation on imprinted plasmonic surfaces. Nat. Commun. 2015, 6, 7337.

(27) Franklin, D.; Frank, R.; Wu, S.-T.; Chanda, D. Actively addressed single pixel fullcolour plasmonic display. Nat. Commun. 2017, 8, 15209.

(28) Bartholomew, R.; Williams, C.; Khan, A.; Bowman, R.; Wilkinson, T. Plasmonic nanohole electrodes for active color tunable liquid crystal transmissive pixels. Opt. Lett. 2017, 42, 2810-2813.

(29) Nasehi, Z.; Nozhat, N. Liquid crystal based tunable plasmonic subtractive color filters. Opt. Commun. 2019, 445, $96-100$.

(30) Xie, Z.-W.; Yang, J.-H.; Vashistha, V.; Lee, W.; Chen, K.-P. Liquid-crystal tunable color filters based on aluminum metasurfaces. Opt. Express 2017, 25, 30764-30770. 
(31) Greybush, N. J.; Charipar, K.; Geldmeier, J. A.; Bauman, S. J.; Johns, P.; Naciri, J.; Charipar, N.; Park, K.; Vaia, R. A.; Fontana, J. Dynamic Plasmonic Pixels. ACS Nano 2019, 13, 3875-3883.

(32) Duempelmann, L.; Gallinet, B.; Novotny, L. Multispectral Imaging with Tunable Plasmonic Filters. ACS Photonics 2017, 4, 236-241.

(33) Wang, H.; Wu, T. X.; Zhu, X.; Wu, S.-T. Correlations between liquid crystal director reorientation and optical response time of a homeotropic cell. J. Appl. Phys. 2004, 95, $5502-5508$.

(34) Aharon, O.; Abdulhalim, I. Liquid crystal Lyot tunable filter with extended free spectral range. Opt. Express 2009, 17, 11426-11433.

(35) Gale, M. G.; Gimkiewicz, C.; Obi, S.; Schnieper, M.; Sochtig, J. Resonant absorption of a chemically sensitive layer based on waveguide gratings. Opt. Lasers Eng. 2005, 43, 373-386.

(36) Liu, Z.; Liu, G.; Wang, Y.; Liu, X.; Tang, C. Silicon-based light absorbers with unique polarization-adjusting effects. Journal of Physics D: Applied Physics 2019, 52, 505109.

(37) Kats, M. A.; Yu, N.; Genevet, P.; Gaburro, Z.; Capasso, F. Effect of radiation damping on the spectral response of plasmonic components. Opt. Express 2011, 19, 21748-21753.

(38) Wu, S.-T. Design of a liquid crystal based tunable electrooptic filter. Appl. Opt. 1989, $28,48-52$.

(39) Yang, D.-K.; Wu, S.-T. Fundamentals of Liquid crystal Devices; Wiley Series in Display technology, 2015.

(40) Harm, W.; Jesacher, A.; Thalhammer, G.; Bernet, S.; Ritsch-Marte, M. How to use a phase-only spatial light modulator as a color display. Opt. Lett. 2015, 40, 581-584. 
(41) Burgos, S. P.; Yokogawa, S.; Atwater, H. A. Color Imaging via Nearest Neighbor Hole Coupling in Plasmonic Color Filters Integrated onto a Complementary Metal-Oxide Semiconductor Image Sensor. ACS Nano 2013, 7, 10038-10047.

(42) Hagen, N. A.; Kudenov, M. Review of snapshot spectral imaging technologies. Opt. Eng. 2013, 52, 090901.

(43) Liddle, J. A.; Gallatin, G. M. Nanomanufacturing: A Perspective. ACS Nano 2016, 10, 2995-3014.

(44) Murthy, S.; Pranov, H.; Feidenhans'l, N. A.; Madsen, J. S.; Hansen, P. E.; Pedersen, H. C.; Taboryski, R. Plasmonic color metasurfaces fabricated by a high speed roll-to-roll method. Nanoscale 2017, 9, 14280-14287.

(45) Xu, T.; Wu, Y.-K.; Luo, X.; Guo, L. J. Plasmonic nanoresonators for high-resolution colour filtering and spectral imaging. Nat. Commun. 2010, 1, 59.

(46) Liu, X.; Fu, G.; Wang, Y.; Pan, P.; Liu, Z. Polarization-Adjusting Ultra-Narrow MultiBand Color Filtering by Dielectric Metamaterials. IEEE Photonics Technology Letters 2016, 28, 979-982.

(47) Luk'yanchuk, B.; Zheludev, N. I.; Maier, S. A.; Halas, N. J.; Nordlander, P.; Giessen, H.; Chong, C. T. The Fano Resonance in Plasmonic Nanostructures and Metamaterials. Nat. Mater. 2010, 9, 707-715.

(48) Liu, Z.; Fu, G.; Liu, X.; Liu, Y.; Tang, L.; Liu, Z.; Liu, G. High-quality multispectral bio-sensing with asymmetric all-dielectric meta-materials. 2017, 50, 165106.

(49) Tseng, L.-T.; Kazazis, D.; Wang, X.; Popescu, C. M.; Robinson, A. P.; Ekinci, Y. Sub-20 nm Si fins with high aspect ratio via pattern transfer using fullerene-based spin-on-carbon hard masks. Microelectron. Eng. 2019, 210, 8 - 13. 
(50) Johnson, P. B.; Christy, R. W. Optical-Constants of Noble-Metals. Phys. Rev. B 1972 , 6,4370 .

(51) Gallinet, B.; Butet, J.; Martin, O. J. F. Numerical methods for nanophotonics: standard problems and future challenges. Laser $\mathcal{E} 3$ Photonics Reviews 2015, 9, 577-603. 


\section{For Table of Contents Use Only}

Title: Electrically tunable multicolored filter using birefringent plasmonic resonators and liquid crystals

Authors: Luc Driencourt, François Federspiel, Dimitrios Kazazis, Li-Ting Tseng, Richard Frantz, Yasin Ekinci, Rolando Ferrini, Benjamin Gallinet
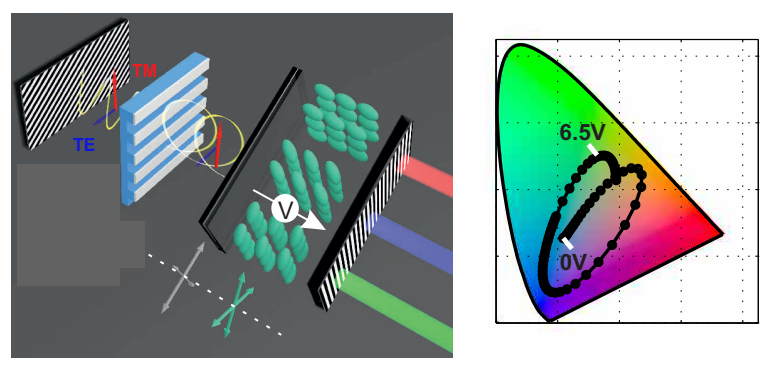

Synopsis: Birefringence-induced colors of plasmonic resonators and a fast switching thin liquid crystal cell are combined in a multicolored electrically tunable filter. In the CIE coordinates, a single filter is able to cover more than $70 \%$ of the color gamut of standard RGB filters by applying a voltage ranging between $2 \mathrm{~V}$ and $6.5 \mathrm{~V}$. 